\title{
Effect of pressure on the phonon properties of europium chalcogenides ${ }^{\dagger}$
}

\author{
U K SAKALLE, P K JHA ${ }^{*}$ and S P SANYAL* \\ Department of Physics, M.V.M., Bhopal 462 001, India \\ Department of Physics, The M.S. University of Baroda, Vadodara 390 002, India \\ *Institute of Physics and Electronics, Bhopal University, Bhopal 462 026, India
}

\begin{abstract}
Lattice vibrational properties of europium chalcogenides have been investigated at high pressure by using a simple lattice dynamical model theory viz. the three-body force rigid ion model (TRIM) which includes long range three-body interaction arising due to charge transfer effects. The dispersion curves for the four Eu-chalcogenides agree reasonably well with the available experimental data. Variation of LO, TO, LA and TA phonons with pressure have also been studied at the symmetry points of the brillouin zone (BZ) for Euchalcogenides for the first time by using a lattice dynamical model theory. We have also calculated the one phonon density of states and compared them with the first order Raman scattering results. The calculation of one phonon density of states for Eu-chalcogenides has also been extended up to the phase transition pressure. We observed a pronounced shift in phonon spectrum as pressure is increased.
\end{abstract}

Keywords. Phonons; high pressure; phase transition.

\section{Introduction}

The europium chalcogenides (EuX: $\mathrm{X}=\mathrm{O}, \mathrm{Se}, \mathrm{S}$ and Te) are the rare earth compounds crystallizing in rock salt structure and in general are magnetic semiconductors. The main interest in EuX compounds, however, has been in their possible applications in magneto optic memories and modulators. Also, much of the interest was shown in these materials in increasing the magnetic ordering temperature with room temperature as final goal to make the practical applications of Eu-chalcogenides realistic (Godowski et al 1991).

It has been shown by experimental as well as theoretical study that these Eu-chalcogenides exhibit various structural as well as magnetic phases with changing temperature and pressure (Hatano et al 1995). These compounds undergo phase transition from $\mathrm{NaCl}(\mathrm{B} 1)$ phase to $\mathrm{CsCl}(\mathrm{B} 2)$ phase with the application of pressure (Jayaraman et al 1974). The structural phase transition pressures for EuO, EuS, EuSe and EuTe are 30-40 GPa, $22 \mathrm{GPa}, 15 \mathrm{GPa}$ and $10 \mathrm{GPa}$, respectively.

In a recent study, Hatano et al (1995) have measured the Raman spectra of EuSe under hydrostatic pressure up to $2.1 \mathrm{GPa}$ at $2 \mathrm{~K}$. They clearly observed that there are distinct spectral changes occurring twice which can be interpreted as magnetic phase transition in EuSe. They have also determined overall features of dispersion curves of LO phonon along [111] direction under high pressure.

\footnotetext{
*Author for correspondence

${ }^{\dagger}$ Paper presented at the 5th IUMRS ICA98, October 1998, Bangalore and respectfully dedicated to Prof. R K Singh on his 60 th birthday.
}

Motivated from this study of Hatano et al, we are impelled to calculate the effect of pressure on different phonon modes of complete group of these compounds by using a simple lattice dynamical model theory, viz. threebody rigid ion model (TRIM).

In the present study, we report the results on the investigations on phonon properties of Eu-chalcogenides by using three-body rigid ion model. Details of the present model theory can be found elsewhere (Jha et al 1995). Section 2 deals with brief discussion on the model theory while $\S 3$ is devoted to results and discussion.

\section{Model theory}

In order to describe the phonon dispersion curves of europium chalcogenides, we use a three-body force rigid ion model, already discussed by Singh (1982). In the present model we consider the many body interactions to arise from the overlap of the electronic charges between the nearest neighbour ions. The dynamical matrix corresponding to TRIM can be written as

$$
D(q)=R+Z m(C+T) Z m,
$$

where $R$ and $C$ are the repulsive and Coulomb matrices, $T$ is three-body force interaction matrix and $\mathrm{Zm}$ the modified ion charge, defined as,

$$
Z m e=+Z e[1+2 n f(r) / z]
$$

where $f(r)$ is the TBI parameter, signifying the amount of overlap. 
The present model has five parameters (four short range and one TBI) which are obtained from macroscopic quantities (e.g. elastic constants, zone centre and zone boundary frequencies). The equations relating to the macroscopic properties with the model parameters are already available in literature (Singh 1982). The input constants and model parameters are presented in tables 1 and 2 , respectively.

\section{Results and discussion}

In the present investigation we have calculated the one phonon density of states for the Eu-chalcogenides. In figures 1 and 2 we have presented the calculated one pho-

Table 1. Input parameters for Eu-chalcogenides.

\begin{tabular}{|c|c|c|c|c|c|}
\hline \multirow[b]{2}{*}{ Solids } & \multirow[b]{2}{*}{$\begin{array}{c}r_{0} \\
(\AA)\end{array}$} & $\mathrm{C}_{11}$ & $\mathrm{C}_{12}$ & $\mathrm{C}_{14}$ & \multirow{2}{*}{$\begin{array}{r}-v_{\mathrm{TO}}(\Gamma) \\
(\mathrm{THz})\end{array}$} \\
\hline & & \multicolumn{3}{|c|}{ (Elastic constants in $10^{12}$ dyne $/ \mathrm{cm}^{2}$ ) } & \\
\hline $\mathrm{EuS}$ & $3 \cdot 09$ & $1 \cdot 1$ & $0 \cdot 1$ & $0 \cdot 2$ & 3 . \\
\hline EuTe & $3 \cdot 292^{\mathrm{a}}$ & 0.936 & $0 \cdot 067$ & $0 \cdot 163^{\mathrm{b}}$ & $3 \cdot 22$ \\
\hline
\end{tabular}

${ }^{\mathrm{a}}$ Onsaka (1977), ${ }^{\mathrm{b}}$ Wyckoff (1963), ${ }^{\mathrm{c}}$ Guntherodt (1974)

Table 2. Output parameters for Eu-chalcogenides obtained by TRIM.

\begin{tabular}{lccccc}
\hline Solids & $A_{12}$ & $B_{12}$ & $A_{22}$ & $B_{22}$ & $Z_{\mathrm{m}}$ \\
\hline EuSe & 19.480 & -4.427 & 1.171 & -0.62 & 1.32 \\
EuTe & 17.160 & -1.027 & 0.876 & -0.421 & 0.974 \\
\hline
\end{tabular}

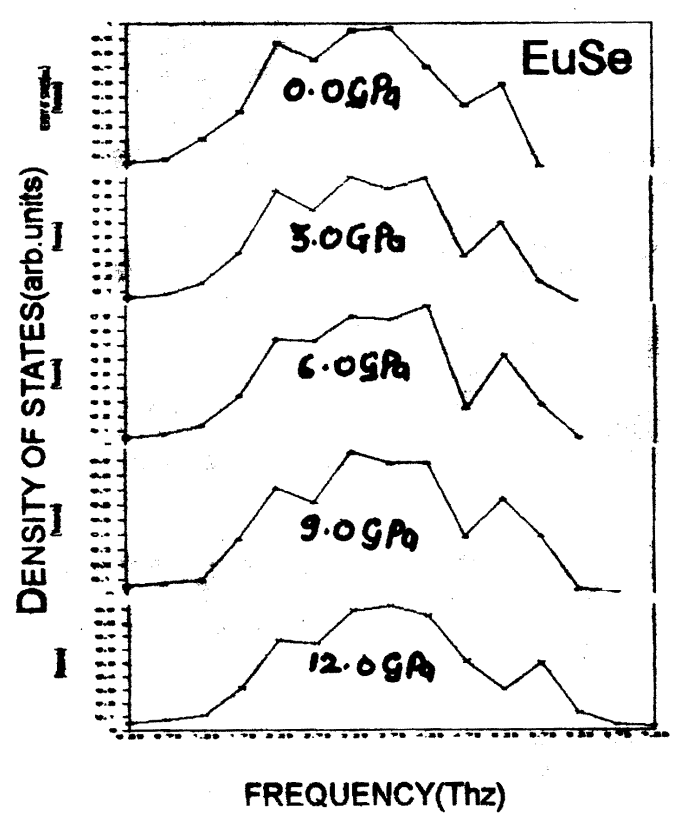

Figure 1. One phonon density of states for EuSe near to the phase transition pressure. non density of states for the EuSe and EuTe near to the phase transition pressure for the first time by using a simple interatomic interaction approach. Since there is no experimental measurement except for EuSe at very low pressure, we could not compare all the OPDOS at high pressure. However, there is good agreement between the experimental Raman measurements of Hatano et al (1995) and OPDOS of present calculation for EuSe at high

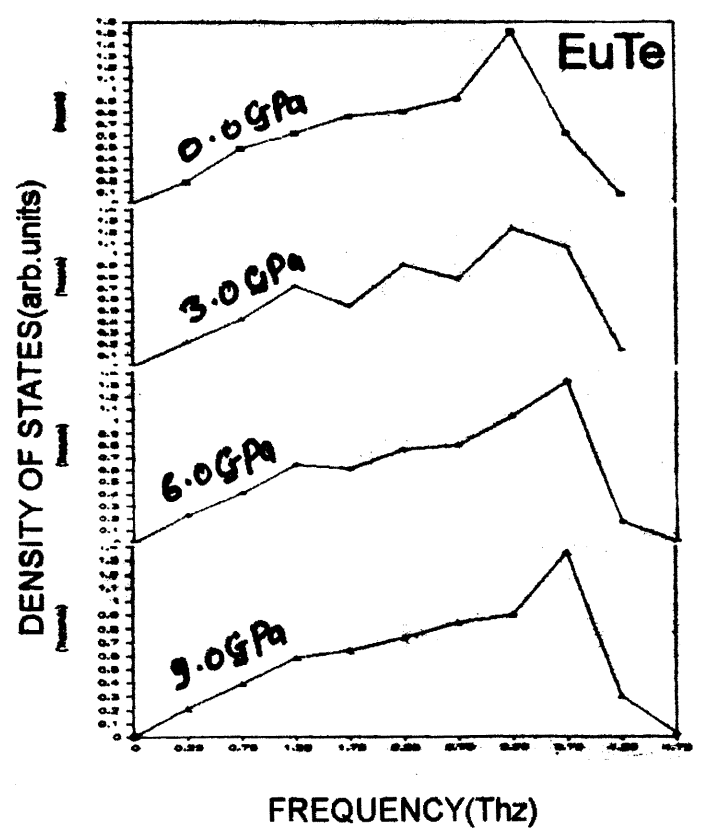

Figure 2. Same as in figure 1 for EuTe.

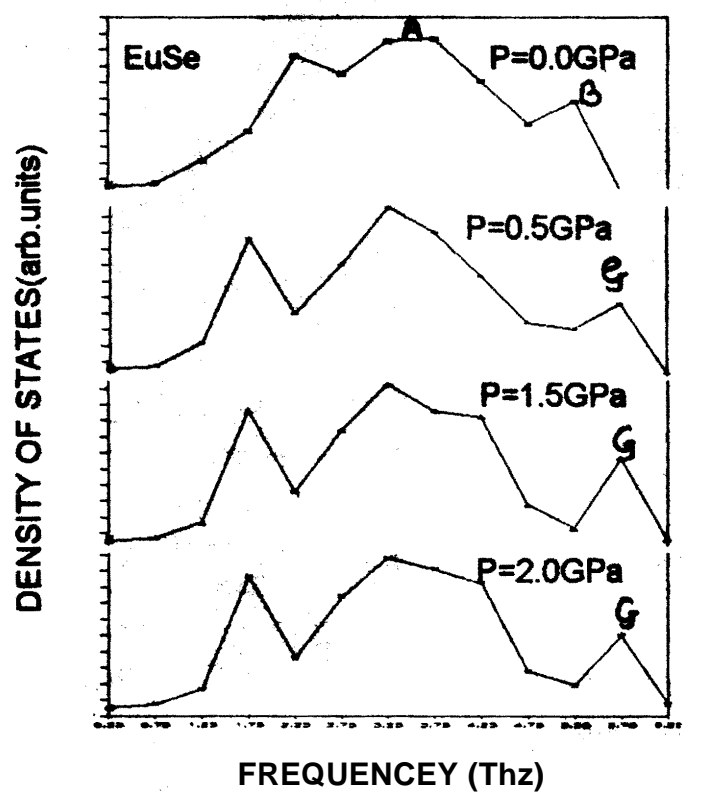

Figure 3. One phonon density of states for EuSe up to 2.0 GPa as suggested by Hatano et al (1995). Peak G is observed from $0.5 \mathrm{GPa}$ to $2 \cdot 0 \mathrm{GPa}$. 


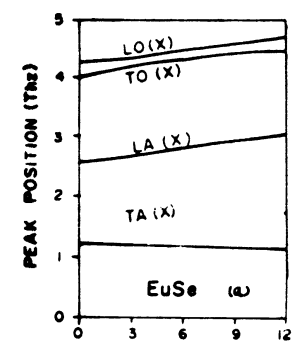

PRESSURE (GPO)

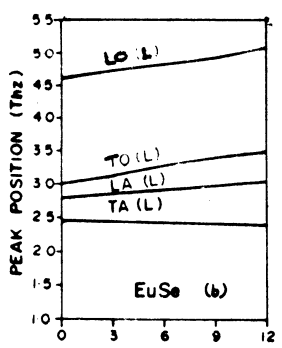

PRESSURE (GPO)

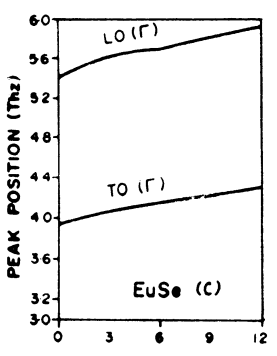

PRESSURE (GPO)

Figure 4. Variation of LO, TO, LA and TA phonons with pressure at the symmetry points of the Brillouin zone (BZ) for EuSe. It follows the similar trend as suggested by Hatano et al (1995).

pressure which have been presented in figure 3. Figures 1 and 2 reveal that as the pressure is increased there is a pronounced shift in the peaks and the maximum magnitude of the OPDOS. This is similar to the characteristics observed in the EuSe by using Raman spectra (Hatano et al 1995). Similar results are obtained for EuO and EuS.

In the absence of the phase transitions the phonon frequencies are expected to increase with the application of hydrostatic pressure. This is because the atoms are brought closer to each other under compression and hence they sit in steeper potential wells. This is termed as normal behaviour of phonons under pressure. However, often one encounters a few phonons which show the opposite behaviour i.e. the frequency of some phonons decreases with increasing pressure. A decrease in frequency implies a reduction in the corresponding force constant which in turn is connected to elastic constants. The phonon which exhibits decrease in frequency with increasing pressure is called a soft phonon as it leads to softening of the lattice. This also points towards instability of the crystal structure against certain atomic vibrations and displacements and often lead to structural phase transition. In figures 4 and 5, we have plotted the varia-
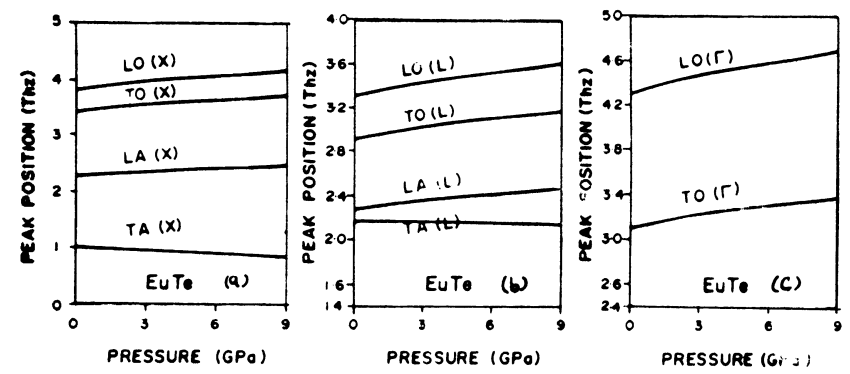

Figure 5. Same as in figure 4 for EuTe.

tion of LO, TO, LA and TA phonons with pressure at the symmetry points of the Brillouin zone (BZ) for EuSe and EuTe as suggested by Hatano et al (1995) in their Raman study. It is evident from these figures that LO, TO and LA phonon frequencies increase with increase in pressure. On the other hand TA phonon is found to exhibit softening. Attempts are still going on to understand the role of TA phonon in driving the phase transition. Similar results are obtained for $\mathrm{EuO}$ and $\mathrm{EuS}$.

In conclusion, we have reported the calculated results on phonon dispersion curves and density of states at high pressure by using a lattice dynamical model viz. the threebody force rigid ion model. We observe a pronounced shift in the phonon spectrum as pressure is increased, similar to the results reported by Raman measurements.

\section{References}

Godowski M et al 1991 in Diluted magnetic semiconductors (ed.) Mukesh Jain (Singapore: World Scientific)

Guntherodt G 1974 Phys. Cond. Matter. 181403

Hatano T et al 1995 J. Phys. Chem. Solids 56531

Jayaraman A et al 1974 Phys. Rev. B9 2513

Jha P K et al 1995 Phys. Rev. 5215898

Onsaka et al 1980 J. Phys. Soc. Jpn. 481269

Singh R K 1982 Phys. Rep. 85259

Wyckoff R W G 1974 in Crystal structure (New York: Wiley) 